\title{
PLUME MODELING AND APPLICATION TO MARS 2001 ODYSSEY AEROBRAKING
}

\author{
Zachary Q. Chavis \\ Joint Institute for Advancement of Flight Sciences \\ The George Washington University \\ NASA Langley Research Center \\ Hampton, VA \\ Richard G. Wilmoth \\ NASA Langley Research Center \\ Hampton, VA
}

\begin{abstract}
$\underline{\text { Abstract }}$
A modified source flow model was used to calculate the plume flowfield from a Mars Odyssey thruster during aerobraking. The source flow model results compared well with previous detailed CFD results for a Mars Global Surveyor thruster. Using an iso-density surface for the Odyssey plume, DSMC simulations were performed to determine the effect the plumes have on the Odyssey aerodynamics. A database was then built to incorporate the plume effects into 6-DOF simulations over a range of attitudes and densities expected during aerobraking. 6-DOF simulations that included the plume effects showed better correlation with flight data than simulations without the plume effects.
\end{abstract}

\section{Introduction}

NASA's 2001 Mars Odyssey was launched on April 7, 2001 and arrived at Mars on October 24, 2001. Odyssey's primary mission is to map the chemical elements and minerals in the Martian surface, look for signs of water and analyze the radiation environment. The Odyssey utilized a technique known as aerobraking to reduce the spacecraft velocity enough to obtain the desired orbit for scientific research. The aerobraking occurred in the upper portions of the Martian atmosphere where the flow over the spacecraft is highly rarefied. During aerobraking, a reaction control system (RCS) was used to maintain the desired spacecraft attitude. The RCS consists of multiple thrusters. When the jets from the thruster firings expand into the vacuum of space or a low-density atmosphere, plumes are created that can have inadvertent effects on the spacecraft. The plumes can impinge on the spacecraft and can interact with the flow around the spacecraft thus altering the aerodynamics. Studies of RCS interactions for Mars Global Surveyor (MGS) found that plume/flowfield interaction effects can be significant ${ }^{1,2}$.

The NASA Langley Research Center (LaRC) provided flight mechanics and atmospheric modeling support for the Mars Odyssey during aerobraking. Part of this support involved providing predictions for each orbit of the aerodynamic behavior of Odyssey. These predictions included a six-degree of freedom (6DOF) analysis of the spacecraft attitude and attitude rates. All known significant forces were modeled in these analyses, including the forces caused by the RCS thrusters. An aerodynamic database was constructed based largely on Direct Simulation Monte Carlo (DSMC) simulations and free-molecular flow calculations. This database was initially constructed to provide the aerodynamic force and moment coefficients of Odyssey over the range of expected atmospheric densities and spacecraft attitudes during aerobraking in the absence of RCS thruster firings. The increments in forces and moments on the spacecraft caused by RCS plume impingement and flow interactions were then determined using a simple "engineering" model of the plume core flow to provide a set of inflow conditions for further DSMC simulations. The purpose of this paper is to provide a description of this simple plume model, describe the implementation of the model for DSMC simulations, and to present results that demonstrate the predicted RCS plume effects on the Odyssey aerodynamics. Validation of the model with a more detailed computational fluid dynamics (CFD) model will be discussed and correlation with flight data will be provided. 


\section{Odyssey Spacecraft}

The Odyssey spacecraft geometry is shown in Figure 1 along with two coordinate systems. The coordinate system with the subscript ' $M$ ' is the spacecraft mechanical coordinate system and the system with the subscript ' $\mathrm{B}$ ' is the POST (Program to Optimize Simulated Trajectories) body frame coordinate system. POST was used for both 3-DOF and 6DOF simulations during LaRC support. The Odyssey RCS thruster arrangement is shown in Figure 2. The thrusters are canted and not aligned with the mechanical axes to provide three-axis control. All of the RCS thrusters are identical. Thruster characteristics are listed in Table 1. Also shown in Figure 2 are four TCM thrusters. These thrusters are primarily used for orbit maneuvers and serve as a backup to the RCS thrusters during aerobraking passes.

\section{Aerobraking Conditions}

As mentioned previously, aerobraking occurred in the upper Martian atmosphere where the flow over the spacecraft is rarefied. The flight conditions were chosen to anticipate the range of densities and attitudes the spacecraft would experience during aerobraking. The densities chosen were; 1, 3, 10, 32 and 100 $\mathrm{kg} / \mathrm{km}^{3}$ which form evenly spaced intervals on a log base ten scale. The range for the attitude chosen was $-20^{\circ}$ to $20^{\circ}$ in both pitch and yaw. Other parameters that were used during the DSMC simulations are listed in Table 2.

\section{DSMC}

DSMC directly models the molecular physics of a gas flow by simulating the flow of particles. To model the rarefied flow of the Martian atmosphere, DAC (DSMC Analysis Code) was used ${ }^{3}$. DAC is able to simulate rarefied gas dynamic environments with complex geometries and flowfield characteristics. DAC also has the ability for parallel implementation, thus greatly reducing the amount of wall-clock time for a simulation. The model shown in Figure 1 represents the actual geometry used for the DSMC simulations. All DSMC simulations were performed using a variable hard sphere model and assumed diffuse wall reflections with full thermal accommodation. The surface temperature was assumed to be constant at 300 $\mathrm{K}$. The DSMC simulations were first run to provide a baseline set of forces and moments without RCS plume effects at the conditions described above. These simulations were then repeated using the plume model described below to provide inflow conditions representing steady state plume flow. Simulations were also performed at complete vacuum conditions to provide the forces and moments resulting from plume impingement without any atmospheric flow over the spacecraft. These forces and moments were converted to coefficient form where appropriate based on a spacecraft reference area of $11.03 \mathrm{~m}^{3}$ and a reference length of $4.74 \mathrm{~m}$.

\section{Plume Model}

RCS plume flows are typically characterized as continuum near the nozzle and then passes though the transition regime before becoming free-molecule flow. In a near vacuum, this expansion occurs within a relatively short distance from the nozzle exit. The plume flow model used in the present study is based on source flow principles and was devised by Woronowicz ${ }^{4}$. Since the model requires the nozzle exit plane properties, it was necessary to determine the internal nozzle flow.

The internal nozzle flow was computed using a CFD program called VNAP ${ }^{5}$. VNAP solves the Navier-Stokes equations using a twostep, predictor-corrector explicit finite-difference method. The 2-D axisymmetric geometry for one of the RCS thrusters was created using the information about the thrusters listed in Table 1. The nozzle geometry upstream of the throat was approximated for the purpose of the CFD simulation. The internal flow was assumed to be laminar.

Source flow models are basically spatial distribution functions for plume flowfield properties derived from conservation of mass and energy ${ }^{4}$. The Woronowicz model divides the exit plane into many point sources. Each point represents a small section of the nozzle exit and has properties based on the local flow in the nozzle. In the current implementation, an arbitrary plume mesh is created downstream of the nozzle exit. The flowfield contributions at each point in the mesh are calculated for each individual point source, and the results are summed to get the total influence of the sources on the flow properties at each mesh point.

The model developed by Woronowicz uses free-molecule theory to describe the flowfield. Assuming that the flow expands radially from each point source, properties at each mesh point can be calculated using the conservation equations. Although the freemolecular description of the flow is not valid in the continuum core of the plume, it has been 
found that the radial expansion assumption gives a reasonably accurate approximation of the spatial variations in plume flow properties at sufficiently large distances from the exit. Furthermore, the free-molecular conservation formulation has been shown to capture much of the functional dependence of these properties on nozzle exit conditions. ${ }^{4}$ Empirically derived corrections can then be made to account for nonlinear behaviors caused by phenomena such as plume shocks and boundary-layer expansion ${ }^{6}$.

The boundary between continuum and transitional flow that is used for DSMC simulation is often determined based on the Bird breakdown parameter ${ }^{7}$, which relates the collision length scale to the gradient length scale for density expansion. This approach was used in the work of Glass ${ }^{8}$, which used a full NavierStokes CFD computation for the continuum portion of the RCS plume for MGS. However, with the current simple source flow model, such an approach is neither practical nor accurate. The source flow theory does not accurately capture the detailed density gradients in the continuum portion of the plume, and computation of the Bird breakdown parameter from the flowfield is likely to produce significant errors. Since the objective of the current work is to capture the first-order plume impingement and flow interaction effects, an alternate scheme was chosen that is expected to satisfy these objectives.

To create a surface for the DAC simulations, an iso-density surface was chosen based on the momentum ratio of the plume to the free stream flow. A momentum ratio of 100 was chosen. Since the plume surface was modeled as an out-gassing surface in DAC, i.e., particles can only flow out of the surface and not into it, the momentum ratio has to be high enough that only a negligible amount of atmosphere particles can penetrate the plume. If the ratio is too low, the amount of particles penetrating the plume will no longer be negligible and error will be introduced into the calculations.

\section{$\underline{\text { Results }}$}

\section{Plume Flowfield}

The nozzle exit properties are given along a line from the centerline of the nozzle to the nozzle wall. Using the assumption that the flow at the exit is symmetric, the solution along a radial line is propagated $360^{\circ}$ about the centerline to form a 2-D exit plane solution.
This solution was input into the source flow model.

The plume number density contour predicted by the source flow model for an Odyssey thruster is shown in Figure 3. Inaccuracies occur near the nozzle exit, but the plume contour lines show the behavior typically expected for a radially expanding flow farther away from the exit. Based on the momentum ratio described previously, a number density of $6.1 * 10^{20}$ molecules $/ \mathrm{m}^{3}$ was used to extract an iso-density surface of the plume. The plume surface corresponding to this density is shown in Figure 4.

\section{MGS Plume Comparison}

Since CFD results were already available for an MGS thruster ${ }^{8}$, which has similar characteristics to the Odyssey thruster, the MGS plume was selected to validate the current source flow model. Again, VNAP was used to calculate the internal nozzle flow, and then the source flow program was used to determine the plume flowfield. Figure 5 compares the number density along the plume centerline for the CFD results and for the source flow program results. The results match up well except in the proximity of the nozzle exit, where the source flow program is not considered accurate. An individual number density contour line $\left(\right.$ nden $=2.0946 \mathrm{E}+22$ molecules $\left./ \mathrm{m}^{3}\right)$ from the CFD results is compared to the same number density contour line from the source flow program results in Figure 6 . The CFD results show a flowfield that is slightly more elongated than the source flow plume flowfield, but overall the two show good correlation.

\section{Baseline Aerodynamies}

DAC simulations were first made with the spacecraft at nominal attitude (zero pitch and yaw) with respect to the free stream velocity for the varying densities with no RCS plumes present. The aerodynamic coefficients predicted by these simulations are included in Table 3 . The moments were shifted to be about the spacecraft center of mass during the midpoint of aerobraking. The center of mass used was; $x=$ $0.0629 \mathrm{~m}, \mathrm{y}=-0.0172 \mathrm{~m}$, and $\mathrm{z}=1.11 \mathrm{~m}$.

One of the first things that can be observed from Table 3 is that the vehicle in the nominal attitude is not at the trim angle. This is evident by the fact that there are nonzero aerodynamic moments on the spacecraft. The coefficient of force in the Y-direction is much larger than the other coefficients because the free 
stream velocity is in the Y-direction. The surface pressure contours on the Odyssey with a free stream density of $100 \mathrm{~kg} / \mathrm{km}^{3}$ are shown in Figure 7. The values from these DSMC simulations will serve as comparisons for later simulations with the plumes added.

\section{$\underline{\text { RCS Studv }}$}

The RCS thrusters for Odyssey may be fired individually or in combinations of two thrusters. However, it was decided to consider just one plume initially, so that the influence of the plume impingement and atmosphere interaction effects could be determined without having to consider possible plume-plume interaction effects. Based on the symmetry of the spacecraft and the RCS thrusters, two cases were chosen, one with the RCS-1 thruster firing and one with the RCS-2 thruster firing. To obtain the maximum possible plume-flowfield interaction effects, these cases were performed at the atmospheric density of $100 \mathrm{~kg} / \mathrm{km}^{3}$. The aerodynamic results of these DSMC simulations are shown in Table 4 . These results represent only aerodynamic and impingement forces and do not include the thrust from the firing. The aerodynamic coefficients from the previous simulations with no plumes at the same atmospheric density are included in the table for comparison. It can be seen that both RCS- 1 and RCS-2 plumes have a small but observable impact on the coefficients of forces and moments on the spacecraft. The surface pressure contours with RCS-1 firing and RCS-2 firing are shown in Figures 8 and 9 respectively. The direct impingement of the plume onto the solar panel is evident for RCS-2. This direct impingement occurs because the RCS-2 nozzle is canted toward the panel.

It is now important to determine how these moment coefficients compare to those caused by the RCS thrust. The forces are not included in this comparison since the attitude is the primary concern of this study. Table 5 shows the moment coefficients caused by aerodynamics only, thrust only, and the combination of the two. From Table 5, it can be seen that the plume impingement and aerodynamic moments are smaller than the thrust moments, but are of a comparable magnitude. Since the plumes induce moments that sometimes oppose the thrust moments, it is important that RCS effects be considered in any 6-DOF simulations of the spacecraft attitude and attitude rates with RCS firings.
RCS interactions during aerobraking are composed of two components, impingement and atmosphere interaction. It is possible to look at the effect of each of these components separately. To do this the same cases can be analyzed with a zero density atmosphere. With no atmosphere, all of the forces and moments on the spacecraft will be a result of plume impingement only. With the assumption that the plume impingement forees do not change even with the addition of an atmosphere, then the plume-flowfield interaction forces and moments can be calculated as the difference between the forces and moments caused by the RCS firing with and without an atmosphere. The assumption that the impingement forces do not change is probably a reasonable assumption since the spacecraft is in the low-density rarefied flow regime for all densities of interest. The breakdown between moments caused by plume impingement and plume-flowfield interaction for RCS-2 is shown in Table 6. The table also includes the moments caused by thrust only to serve as a comparison of magnitudes. The plume impingement moments are larger in magnitude than the plume-flowfield interaction moments but in opposite directions for the $\mathrm{x}$ and $\mathrm{z}$ components. It should be noted that the combined moments caused by the RCS-2 firing are all in the opposite direction of the thrust. The thrust has the larger magnitude, but because of the RCS-2 aerodynamic effects the thrusters effectiveness could be reduced.

\section{RCS Database Construction}

The task shifts to incorporating the plume effects into a POST 6-DOF simulation of Odyssey during aerobraking. For the simulation, specific combinations of RCS thrusters fire when the Odyssey attitude or attitude rates exceed certain critical values. A database had already been constructed for the POST 6-DOF simulations to give the aerodynamic coefficients for the Odyssey as a function of attitude and density in the absence of RCS firings. A new database was constructed that compliments the original database and includes the change in aerodynamic coefficients as a result of the RCS firings over a range of attitudes and atmospheric densities.

A procedure was developed that incorporates the RCS aerodynamic effects for POST simulations. The procedure requires the attitude, in POST coordinate frame, density and thruster on/off as input. The database is then called to determine the change in aerodynamic 
coefficients. Linear interpolation is used to interpolate coefficients as a function of attitude and density.

To complete the database in the timeframe needed, some simplifying assumptions were made. The first major assumption was that superposition holds. To test this assumption, three situations were looked at, superposition with RCS-2 and RCS-3, with RCS-1 and RCS-3, and with RCS-1 and RCS-2. Refer to Table 7 for the results (in dimensional form). For example purposes, the focus will be on the superposition using RCS-2 and RCS-3. At nominal attitude and a density of $100 \mathrm{~kg} / \mathrm{km}^{3}$, four simulations were performed. The first simulation was with no plumes. Then two simulations were performed, one with only RCS2 firing and another with only RCS-3 firing. With these three simulations, the change in coefficients caused by RCS-2 and RCS-3 were determined separately. Based on the assumption of superposition, a simulation with both RCS-2 and RCS-3 firings should give the same change in coefficients as just adding the two separate changes in coefficients. The two separate sets of delta coefficients were then added and compared to the increments obtained from a DSMC simulation with both RCS-2 and RCS-3 firings simultaneously. The difference between the actual values and the superpositionapproximated values are shown in column 8 of Table 7. The superposition assumption for this case appears to be reasonably good, with errors less than $10 \%$ of the total moment. It is also shown to be a good assumption for the other two cases as well. Superposition eliminates the need to perform DSMC simulations for combinations of thruster firings, including thruster firings that result in plume-plume interations.

The next major assumption is that the change in coefficients caused by RCS-3 and RCS-4 firings can be determined from the change in coefficients caused by RCS-2 and RCS-1 firings respectively. This assumption is made possible by the symmetry of the spacecraft.

Additional DSMC simulations were performed to develop a database for the nominal attitude at each density. Then an interpolation scheme was developed to interpolate values between densities. It is assumed that the RCS increments for different attitudes will vary with density in a similar manner. Additional DSMC simulations were then performed at attitudes of \pm $15^{\circ}$ in pitch and yaw to provide sufficient data to define variations in the RCS increments with attitude. The database was enhanced utilizing a curve fitting technique. This technique was used to expand the database into coefficients at fivedegree increments with respect to pitch and yaw from $-20^{\circ}$ to $20^{\circ}$ for the full range of densities.

\section{6-DOF Simulations}

A POST 6-DOF simulation was compared to flight data for typical densities experienced during an aerobraking pass. Orbit 24 was chosen to represent the average pass. Two 6-DOF simulations were performed for this orbit, one with the RCS plume effects subroutine active and one with it inactive. The attitude rates for this pass are shown in Figure 10. For the roll rate, there are significant differences between simulation and flight data and including the RCS plume effects subroutine improves the prediction by about a factor of two. For the pitch rate and the yaw rate, there is more reasonable agreement between simulation and flight data and RCS firings have little effect until the end of the pass.

The attitude for the pass is shown in Figure 11. Here, there is a noticeable effect on the simulation results caused by the inclusion of the RCS plume effects model. The model greatly improves the predictions after periapsis in roll, pitch, and yaw. Overall the RCS model allows the POST 6-DOF simulations to match more closely with the actual Odyssey flight data. Orbit 24 represents the typical aerobraking pass and a similar conclusion can be drawn for most other passes.

\section{Summary}

A source flow program was used to determine the plume flowfield for the Odyssey RCS thrusters. The program was also used to determine the plume flowfield for an MGS thruster and compared to the results obtained using a detailed CFD analysis. The results compared favorably. It was concluded that the source flow code gave reasonably accurate results with minimal computational time required.

A study of the RCS effects on the Odyssey aerodynamics was then performed. Assumptions were made based on the symmetry of the spacecraft and superposition that reduced the number of DSMC simulations necessary. The assumptions proved to be reasonable. An RCS database of the change in aerodynamic coefficients caused by the plumes was constructed through a series of DSMC simulations coupled with a curve fitting technique. Inclusion of the RCS plume effects in POST 6-DOF simulations proved to significantly 
increase the accuracy of the predictions in roll rate and the spacecraft attitude.

\section{Acknowledgements}

The authors would like to thank Chris Glass of the Langley Research Center for use of his CFD results, Michael Woronowicz of the Goddard Spaceflight Center for use of his source flow model, and Jill Hanna of the Langley Research center for use of her POST results.

Research done by Zachary Q. Chavis is a portion of a Master of Science Thesis with The George Washington University.

\section{References}

${ }^{1}$ Rault, D.F., "RCS Plume Effect of Spacecraft Aerodynamics During Aerobraking Maneuver," $20^{\text {th }}$ International Symposium on Rarefied Gas Dynamics, Institute of Mechanics, CAS, Beijing, China, August 1996.

${ }^{2}$ Shane, R.W., and Tolson, R.H.,

"Aerothermodynamics of the Mars Global Surveyor Spacecraft," M.S. Thesis, George Washington University, March 1998.

${ }^{3}$ LeBeau, G.J., and Lumpkin III, F.E., "Application Highlights of the DSMC Analy sis Code (DAC) Software for Simulating Rarefied
Flows," Computer Methods in Applied Mechanics and Engineering, Vol. 191, Issue 6-7, 2001, pp. 595-609

\footnotetext{
${ }^{4}$ Woronowicz, M.S., and Rault, D.F.G., "On Plume Flowfield Analysis And Simulation
} Techniques," AIAA Paper No. 94-2048, AIAA/ASME $6^{\text {th }}$ Joint Thermophysics and Heat Transfer Conference, Colorado Springs, Colorado, June 1994.

${ }^{5}$ Cline, M.C., "VNAP: A Computer Program for Computation of Two-Dimensional, TimeDependent Compressible, Viscous, Internal Flow, Los Alamos Scientific Laboratory, LA7326, Los Alamos, New Mexico, Nov. 1978.

6 "Model for Prediction Orbiter PRCS Plume Impingement Loads and Heating," JSC-26507, NASA Johnson Space Center, June 1995.

${ }^{7}$ Bird, G.A., Molecular Gas Dynamics and the Direct Simulation of Gas Flows, Clarendon Press, Oxford, 1994.

${ }^{8}$ Glass, C.E., "Numerical Study of Rarefied Hypersonic Flow Interacting With a Continuum Jet," NASA Langley Research Center, NASA/TP-2000-210551, Hampton, Virginia, November 2000 .

Table 1. Odyssey RCS Nozzle Specifications

\begin{tabular}{|l|l|}
\hline Thrust & $0.8896 \mathrm{~N}$ \\
\hline Exit Radius & $0.29 \mathrm{~cm}$ \\
\hline Area Ratio & $100: 1$ \\
\hline Chamber Pressure & $2.034 \mathrm{MPa}$ \\
\hline Chamber Temperature & $1166.7 \mathrm{~K}$ \\
\hline Exit Half Angle & 15 degrees \\
\hline Exit Mach Number & 6.41 \\
\hline
\end{tabular}

Table 2. DSMC Parameters

\begin{tabular}{|c|c|}
\hline Free Stream Velocity & $4811 \mathrm{~m} / \mathrm{s}$ \\
\hline Translational Temperature & $144.77 \mathrm{~K}$ \\
\hline CO2 Mole Fraction & 0.9537 \\
\hline N2 Mole Fraction & 0.0463 \\
\hline
\end{tabular}


Table 3. Aerodynamic Coefficients about CM for Mars Odyssey With Varying Densities

\begin{tabular}{|c|c|c|c|c|c|}
\hline & 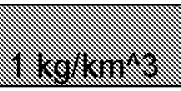 & 3.28213 & 10.64 & $32 / 6 / 4213$ & $y^{2 / 28}$ \\
\hline \&is & 0.0116 & 0.0366 & 0.1157 & 0.3659 & 1.1569 \\
\hline 8 & -0.0002 & 0.0001 & 0.0005 & 0.0021 & 0.0059 \\
\hline 8 & 2.1322 & 2.1046 & 2.0588 & 2.0203 & 1.9457 \\
\hline 8 & -0.0041 & -0.0061 & -0.0106 & -0.0182 & -0.0286 \\
\hline 818 & 0.0048 & 0.0047 & 0.0044 & 0.0043 & 0.0043 \\
\hline 818 & -0.0005 & -0.0008 & -0.0013 & -0.0012 & -0.0014 \\
\hline 1.2 & 0.0301 & 0.0300 & 0.0284 & 0.0277 & 0.0255 \\
\hline
\end{tabular}

Table 4. Aerodynamic Coefficients about $\mathrm{CM}$ for Mars Odyssey With Plumes, Density $=100 \mathrm{~kg} / \mathrm{km}^{3}$

\begin{tabular}{|l|l|l|}
\hline & & \\
0.0059 & 0.0054 & 0.0070 \\
1.9457 & 1.9161 & 1.9577 \\
0.0286 & -0.0163 & -0.0199 \\
\hline 0.0043 & 0.0017 & 0.0044 \\
0.0014 & -0.0093 & -0.0071 \\
0.0255 & 0.0160 & 0.0326 \\
\hline
\end{tabular}

Table 5. Breakdown of RCS Thrust and Aerodynamic Forces, Density $=100 \mathrm{~kg} / \mathrm{km}^{3}$

Moment Coefficients about CM, aerodynamic forces only

\begin{tabular}{|c|c|c|c|}
\hline & 18 & 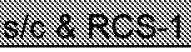 & $5 / C_{1}, 105$ \\
\hline C. or. & 0.0043 & 0.0017 & 0.0044 \\
\hline C. & -0.0014 & -0.0093 & -0.0071 \\
\hline dis: & 0.0255 & 0.0160 & 0.0326 \\
\hline
\end{tabular}

Moment Coefficients about CM, thrust forces only

\begin{tabular}{|c|c|c|c|}
\hline & 58 & 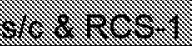 & $2 / 0,2=8$ \\
\hline C. & 0.0000 & 0.0037 & -0.0037 \\
\hline C. & 0.0000 & 0.0132 & 0.0132 \\
\hline 18 & 0.0000 & 0.0128 & -0.0123 \\
\hline
\end{tabular}

Moment Coefficients about $\mathrm{CM}$, thrust and aerodynamic forces

\begin{tabular}{|rrrr}
\hline & 0.0043 & 0.0053 & 0.0007 \\
0.0014 & 0.0039 & 0.0062 \\
0.0255 & 0.0288 & 0.0203 \\
\hline
\end{tabular}

Table 6. RCS-2 Plume Impingement and Flowfield Interaction Effects about CM, Density $=100 \mathrm{~km} / \mathrm{kg}^{3}$

\begin{tabular}{|c|c|c|c|c|c|c|}
\hline & 2 & 3 & 8 & 8 & 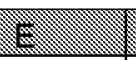 & 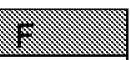 \\
\hline (1) & 0.0043 & 0.0044 & 0.0001 & 0.0033 & -0.0032 & -0.0037 \\
\hline (6) & -0.0014 & -0.0071 & -0.0057 & -0.0032 & -0.0025 & 0.0132 \\
\hline Q.12: & 0.0255 & 0.0326 & 0.0071 & 0.0101 & -0.0030 & -0.0123 \\
\hline
\end{tabular}

$\mathrm{A}=$ Aerodynamic moments on Odyssey, no RCS

$\mathrm{B}=$ Aerodynamic moments on Odyssey, with RCS-2

$\mathrm{C}=$ Total RCS-2 interaction $(\mathrm{B}-\mathrm{A})$

$\mathrm{D}=\mathrm{RCS}-2$ impingement

$\mathrm{E}=\mathrm{RCS}-2$ flowfield interaction (C-D)

$\mathrm{F}=\mathrm{RCS}-2$ thrust only 
Table 7. Superposition of RCS Effects for Multiple Thruster Firings about the CM, Density $=100$ $\mathbf{k m} / \mathbf{k g}^{3}$

RSC-2 \& RCS-3

\begin{tabular}{|c|c|c|c|c|c|c|c|c|c|}
\hline & 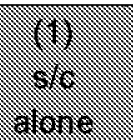 & 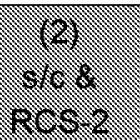 & $\begin{array}{ll}3 & 3 \\
3 & 3 \\
3 & 3\end{array}$ & $\begin{array}{l}3 \\
3 \\
2\end{array}$ & 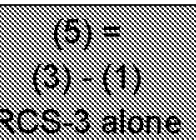 & 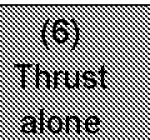 & 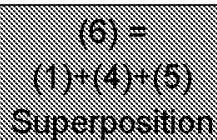 & 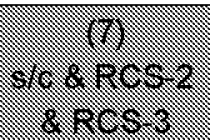 & 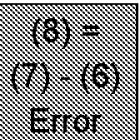 \\
\hline 118 & 0.2606 & 0.2659 & 0.2445 & 0.0053 & -0.0160 & -0.4571 & 0.2499 & 0.2787 & 0.0288 \\
\hline 18 & -0.0826 & -0.4289 & 0.2192 & -0.3463 & 0.3019 & 0.0194 & -0.1271 & -0.1265 & 0.0006 \\
\hline 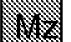 & 1.5443 & 1.9722 & 1.1573 & 0.4279 & -0.3870 & -0.0466 & 1.5852 & 1.5048 & -0.0804 \\
\hline
\end{tabular}

RSC-1 \& RCS-3

\begin{tabular}{|c|c|c|c|c|c|c|c|c|c|}
\hline & 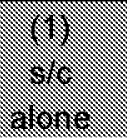 & 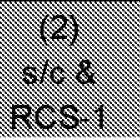 & 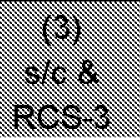 & $\begin{array}{l}1 \\
1 \\
1\end{array}$ & 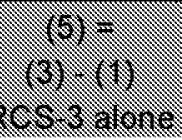 & 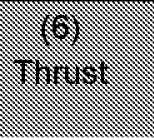 & 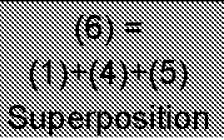 & 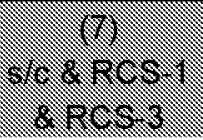 & 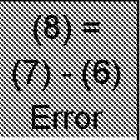 \\
\hline 38 & 0.2606 & 0.1002 & 0.2445 & -0.1603 & -0.0160 & -0.0053 & 0.0842 & 0.1104 & 0.0262 \\
\hline (b) & -0.0826 & -0.4939 & 0.2192 & -0.4113 & 0.3019 & 0.0194 & -0.1921 & -0.2273 & -0.0353 \\
\hline $\mathrm{N}_{3}$ & 1.5443 & 0.9685 & 1.1573 & -0.5758 & -0.3870 & 1.4734 & 0.5816 & 0.5757 & -0.0059 \\
\hline
\end{tabular}

RSC-1 \& RCS-2

\begin{tabular}{|c|c|c|c|c|c|c|c|c|c|}
\hline & 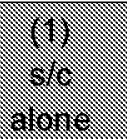 & 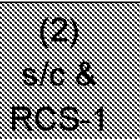 & 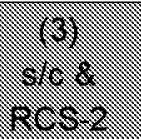 & 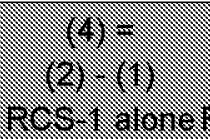 & 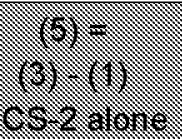 & 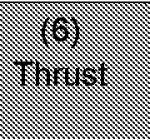 & 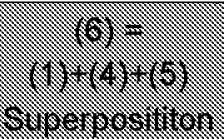 & 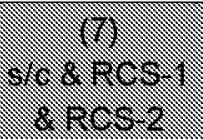 & 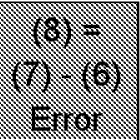 \\
\hline (1) & 0.2606 & 0.1002 & 0.2659 & -0.1603 & 0.0053 & -0.0053 & 0.1056 & 0.0660 & -0.0396 \\
\hline 18 & -0.0826 & -0.4939 & -0.4289 & -0.4113 & -0.3463 & 1.6026 & -0.8402 & -0.6931 & 0.1472 \\
\hline 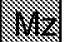 & 1.5443 & 0.9685 & 1.9722 & -0.5758 & 0.4279 & 0.0273 & 1.3965 & 1.5190 & 0.1225 \\
\hline
\end{tabular}

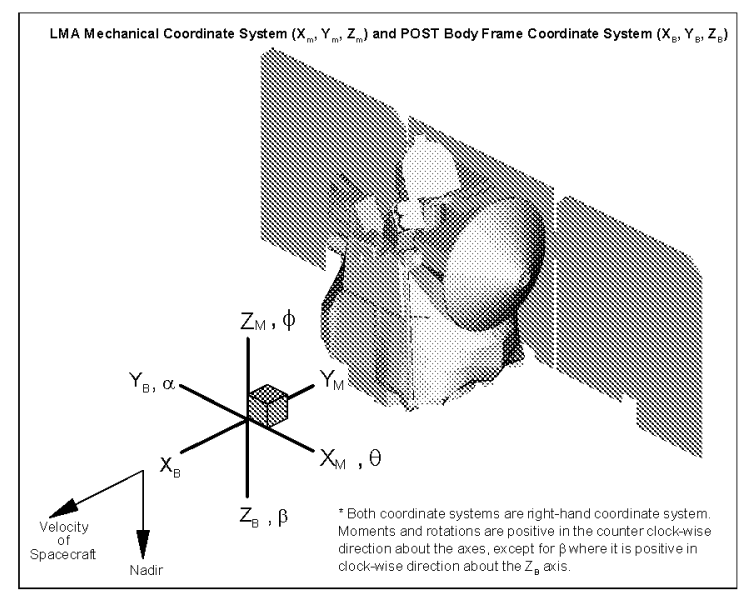

Figure 1. Odyssey Coordinate Frames 


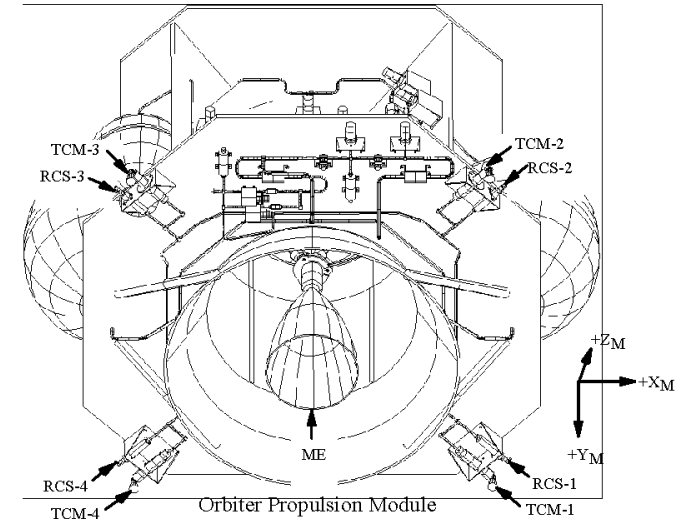

Figure 2. Odyssey Thruster Arrangement

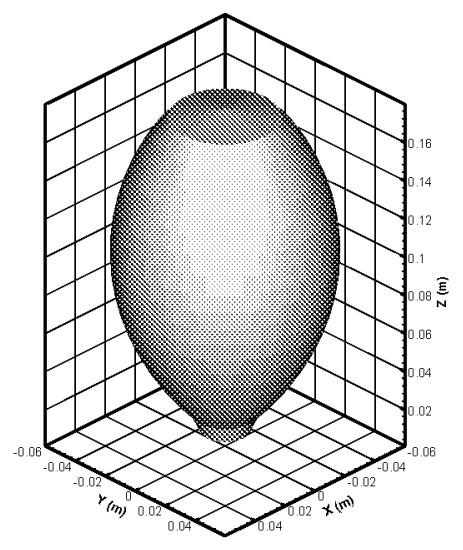

Figure 4. Iso-Density Plume Surface for an Odyssey thruster, Momentum Ratio $=100, \mathrm{n}=6.1^{*} 10^{20} / \mathrm{m}^{3}$

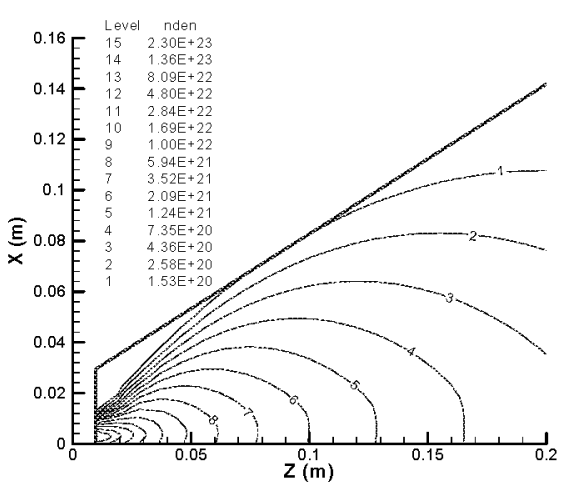

Figure 3. Plume number density contours for an Ody ssey thruster

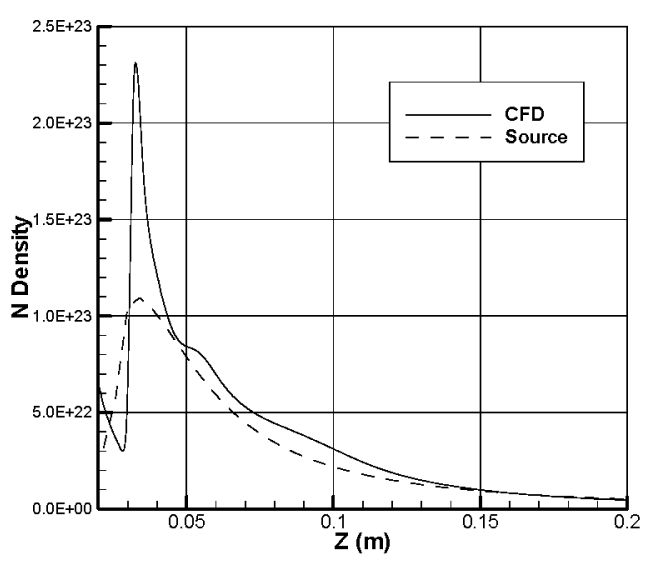

Figure 5. Comparison of plume centerline number density between the source flow code and CFD for MGS plume 


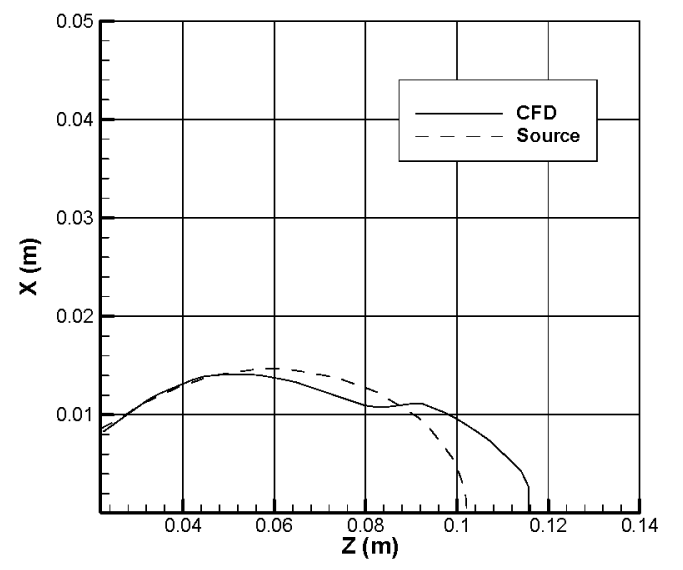

Figure 6. Plume contour comparison between the source flow code and CFD for MGS, number density, nden = $2.0946 \mathrm{E}+22$ molecules $/ \mathrm{m}^{3}$

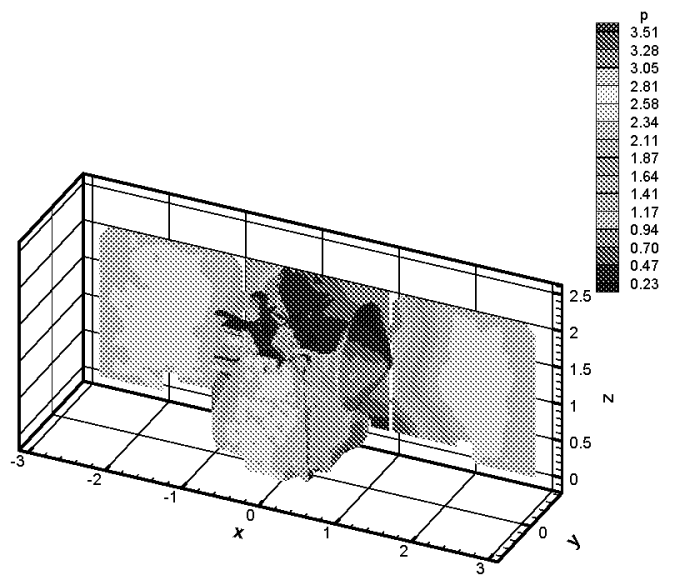

Figure 8. Odyssey Pressure Contour, RCS-1 Plume, $\rho_{\infty}=100 \mathrm{~kg} / \mathrm{km}^{3}$

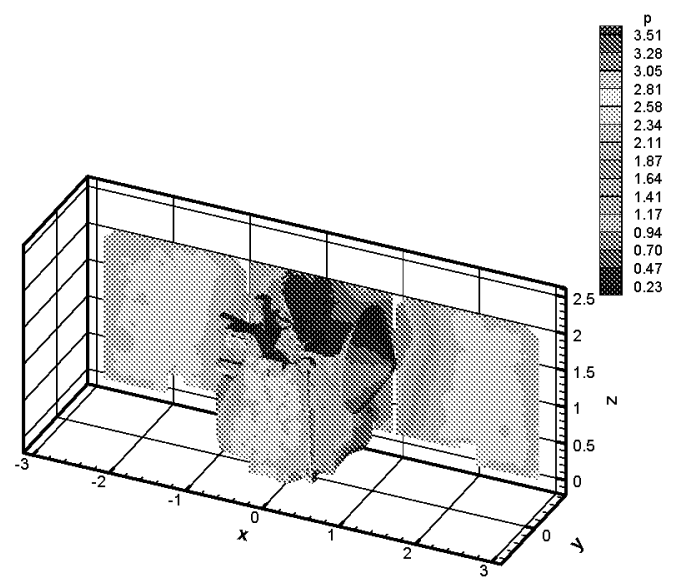

Figure 7. Odyssey Pressure Contour No Plumes, $\rho_{\infty}=100$

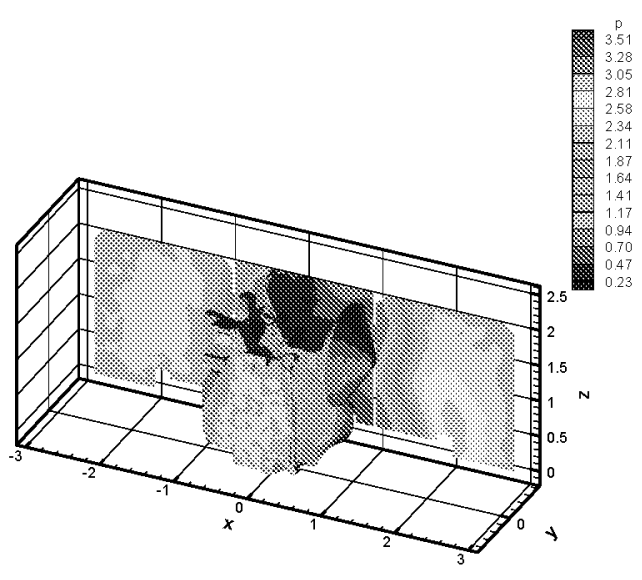

Figure 9. Odyssey Pressure Contour RCS-2 Plume, $\rho_{\infty}=100 \mathrm{~kg} / \mathrm{km}^{3}$ 

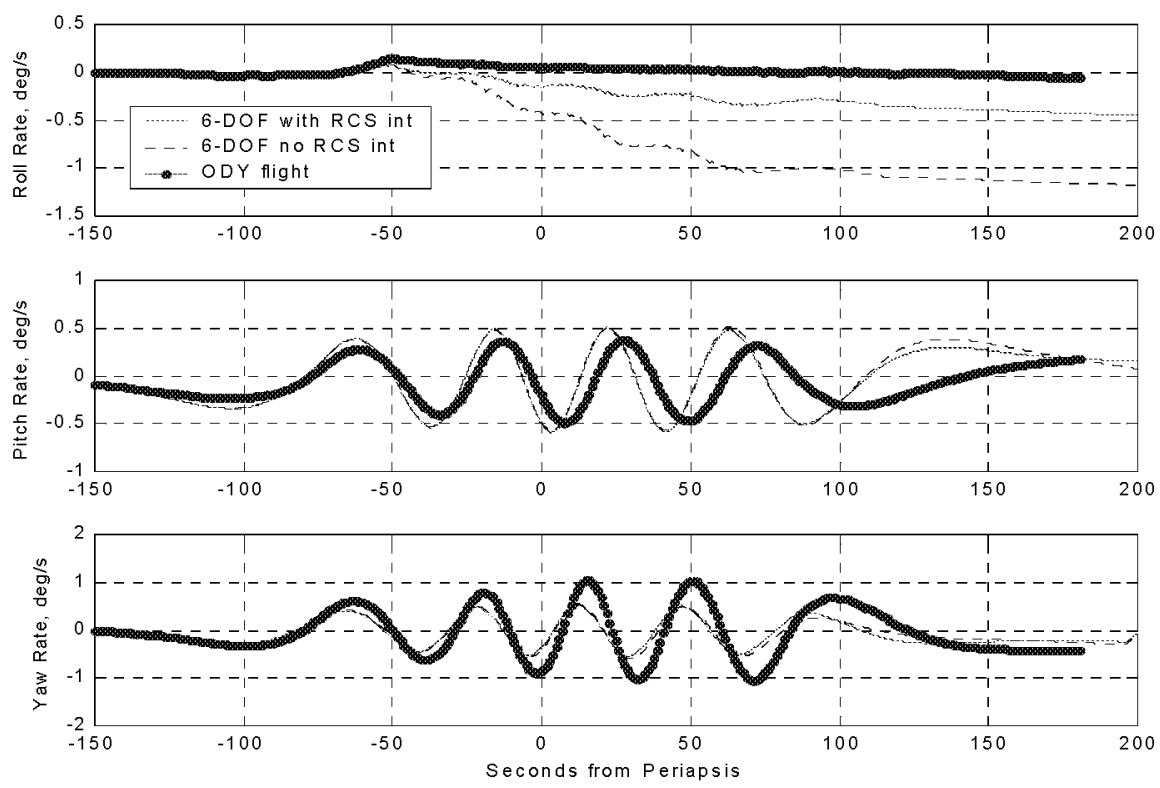

Figure 10. Attitude rates comparison of 6-DOF simulation to Odyssey flight data, orbit 24, RCS model active
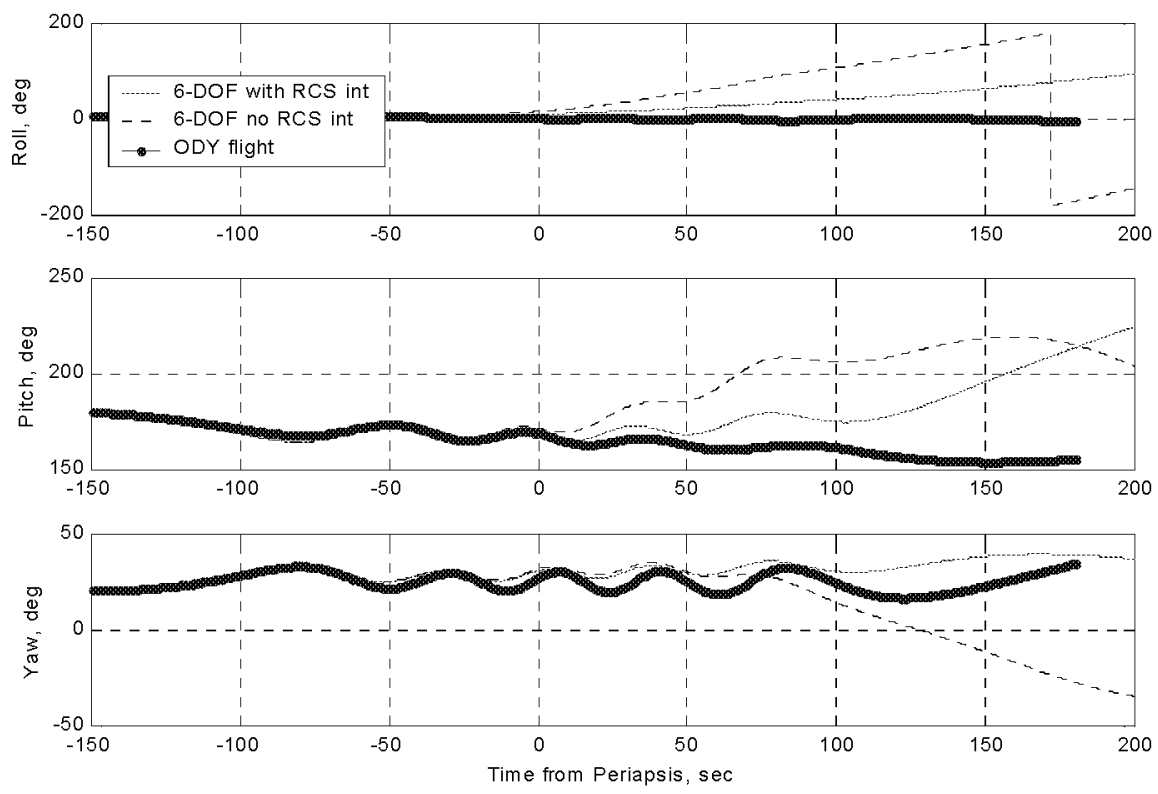

Figure 11. Attitude comparison of 6-DOF simulation to Odyssey flight data, orbit 24, RCS model active 